\title{
Shi-Jiang Li, PhD, Recipient of 2017 Alzheimer Award
}

The Journal of Alzheimer's Disease (JAD) is pleased to announce that Shi-Jiang Li, PhD, Professor of Biophysics, Radiology, and Psychiatry and Behavioral Medicine at the Medical College of Wisconsin, has been chosen as the recipient of the 2016 Alzheimer Award presented by the journal in recognition of his outstanding work on shedding light on the link between preclinical AD status and symptomatic onset that can be applied to accurately identify progressive AD trajectories and quantify disease severity on an individual patient basis.

The winning paper is "Staging Alzheimer's Disease Risk by Sequencing Brain Function and Structure, Cerebrospinal Fluid, and Cognition Biomarkers" [1].

This yearly award is made possible by support from IOS Press.

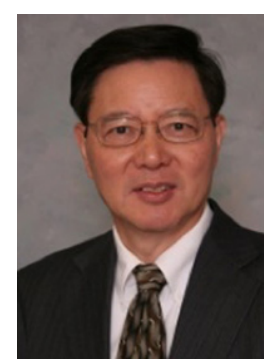

Dr. Shi-Jiang Li graduated from the Department of Electronic Engineering from Tsinghua University, Beijing, China, in 1970; completed his master's program training at the Institute of Biophysics, Chinese Academy of Sciences, in 1980; and received his $\mathrm{PhD}$ in biochemistry from the Ohio State University in 1985. He completed his postdoctoral fellowship training at the Johns Hopkins University, School of Medicine, in 1988. He joined the faculty of the Medical College of Wisconsin in 1988. He is currently Professor of Biophysics, Radiology, and Psychiatry and Behavioral Medicine. He published the first article to evaluate a resting-state fMRI index as a potential biomarker for Alzheimer's disease [2]. From 2009 to 2016, he served as the Director of the Center for Imaging Research at the Medical College of Wisconsin. Since 1990, he has been continuously funded by the NIH and private foundation grants. He is a mentor and sponsor or co-sponsor of 21 current and former NIH career development awards trainees, postdoctoral fellows, and $\mathrm{PhD}$ students. In collaboration with his colleagues, his research focuses on the development of cutting-edge MRI technologies to study Alzheimer's disease, drugs abuse, and human consciousness and translate the findings to clinical applications. He has published more than 110 articles, books, and chapters.

Importance of Published Article

Alzheimer's disease (AD) is a complex, multifactorial disease. It has been difficult to develop a biomarker to closely link preclinical $\mathrm{AD}$ status with continuous disease development to accurately predict progressive $\mathrm{AD}$ trajectories, given the condition that the insidious disease onset often takes decades. Built on the advancement of the event-based probabilistic model in $\mathrm{AD}$ research [3], we integrated the 10 available AD biomarkers to calibrate the temporal sequence of events that occur during the AD developmental continuum. Our findings in the article "Staging Alzheimer's Disease Risk by Sequencing Brain Function and Structure, Cerebrospinal Fluid, and Cognition Biomarkers" [1] demonstrated that the abnormal functional connectivity events in the hippocampus network and the default mode network are the "upstream" factors that occur before the abnormal $\mathrm{A} \beta$ and p-tau level events occur in the cerebrospinal fluid, supporting the network dysfunction hypothesis of AD. Using the 10 sequential numeric scores, we created an index-termed the characterizing Alzheimer's disease risk events, or "CARE," index - to characterize risks associated with AD stages and quantify disease severity on an individual subject basis. The CARE index constructed the main framework for biology-based AD stages and demonstrated that clinically diagnosed mild cognitive impairment subjects are biologically heterogeneous and are distributed across the $\mathrm{AD}$ continuum. We believe the CARE index will have wide clinical applications. If the 
temporal course of disease progression is consistent with biomarker-defined events in individuals, we will have a crucial window of opportunity to intervene with disease-modifying therapy. The CARE index will facilitate clinical trials by selecting the right segmentation of patient populations based on their disease stages to enrich the response rate and statistical power; it can also be used to monitor and evaluate treatment efficacy through changes in stage status in individual subjects.

\section{REFERENCES}

[1] Chen G, Shu H, Chen G, Ward BD, Antuono PG, Zhang Z, Li SJ, Alzheimer's Disease Neuroimaging Initiative (2016) Staging Alzheimer's disease risk by sequencing brain function and structure, cerebrospinal fluid, and cognition biomarkers. J Alzheimers Dis 54, 983-993.

[2] Li SJ, Li Z, Wu G, Zhang MJ, Franczak M, Antuono PG (2002) Alzheimer Disease: Evaluation of a functional MR imaging index as a marker. Radiology 225, 253-259.

[3] Young AL, Oxtoby NP, Daga P, Cash DM, Fox NC, Ourselin S, Schott JM, Alexander DC, Alzheimer's Disease Neuroimaging Initiative (2014) A data-driven model of biomarker changes in sporadic Alzheimer's disease. Brain 137(Pt 9), 2564-2577. 